Documentation et bibliothèques

\title{
La place des bibliothèques dans les revues de littérature de jeunesse
}

\section{Libraries as Portrayed in Childrens' Literature Periodicals El lugar de las bibliotecas en las revistas de literatura juvenil}

\section{Suzanne Pouliot}

Volume 44, numéro 4, octobre-décembre 1998

La lecture publique au Québec : évolution et discours

URI : https://id.erudit.org/iderudit/1032825ar

DOI : https://doi.org/10.7202/1032825ar

Aller au sommaire du numéro

Éditeur(s)

Association pour l'avancement des sciences et des techniques de la documentation (ASTED)

ISSN

0315-2340 (imprimé)

2291-8949 (numérique)

Découvrir la revue

Citer cet article

Pouliot, S. (1998). La place des bibliothèques dans les revues de littérature de jeunesse. Documentation et bibliothèques, 44(4), 177-182.

https://doi.org/10.7202/1032825ar
Résumé de l'article

Depuis près de trente ans, deux revues québécoises de littérature d'enfance et de jeunesse, Lurelu et Des livres et des jeunes, ont contribué à souligner, à décrire et à faire connaître à un public spécialisé (bibliothécaires, libraires, enseignants, étudiants), le travail réalisé par les bibliothèques scolaires et publiques pour accroître la lecture publique des jeunes. Les périodiques étudiés consacrent un large espace éditorial aux activités et aux techniques d'animation réalisées autant par des bibliothécaires chevronnées que par des parents bénévoles, des enseignants et des enseignantes.
Tous droits réservés (c) Association pour l'avancement des sciences et des techniques de la documentation (ASTED), 1998
Ce document est protégé par la loi sur le droit d'auteur. L’utilisation des services d'Érudit (y compris la reproduction) est assujettie à sa politique d'utilisation que vous pouvez consulter en ligne.

https://apropos.erudit.org/fr/usagers/politique-dutilisation/ 


\title{
La place des bibliothèques dans les revues de littérature de jeunesse
}

\author{
Suzanne Pouliot \\ Université de Sherbrooke
}

Depuis près de trente ans, deux revues québécoises de littérature d'enfance et de jeunesse, Lurelu et Des livres et des jeunes, ont contribué à souligner, à décrire et à faire connaître à un public spécialisé (bibliothécaires, libraires, enseignants, étudiants), le travail réalisé par les bibliothèques scolaires et publiques pour accroître la lecture publique des jeunes. Les périodiques étudiés consacrent un large espace éditorial aux activités et aux techniques d'animation réalisées autant par des bibliothécaires chevronnées que par des parents bénévoles, des enseignants et des enseignantes

\section{Libraries as Portrayed in Childrens' Literature Periodicals}

During the past thirty years, two childrens' and young adults' literature journals, Lurelu and Des livres et des jeunes, identified, described and promoted the work of school and public libraries in encouraging reading among youth; their intended audience was specialised (librarians, booksellers, teachers, and students). The two journals devoted considerable editorial space to storytelling activities and techniques by experienced librarians, parents, and teachers.

\section{El lugar de las bibliotecas en las revistas de literatura juve- nil}

Desde hace casi treinta años, dos revistas quebequenses de literatura infantil y juvenil, Lurelu y Des livres et des jeunes, han contribuido a poner de relieve, describir y dar a conocer a un público especializado (bibliotecarios, libreros, maestros, estudiantes) el trabajo realizado por las bibliotecas escolares y públicas para aumentar la lectura pública de los jóvenes. Los periódicos consagran un gran espacio editorial a las actividades y técnicas de animación realizadas tanto por los bibliotecarios experimentados como por los padres que trabajan en forma benévola en las bibliotecas y los maestros.
Depuis bientôt trente ans, préoccupées par la lecture publique, deux revues professionnelles de littérature d'enfance et de jeunesse publiées au Québec ont contribué à décrire le travail réalisé auprès des jeunes par les bibliothèques publiques et scolaires et, à l'occasion, par leur corollaire, le coin de lecture. La revue Lurelu (1977- ）, consacrée exclusivement à la littérature québécoise d'enfance et de jeunesse, et la revue Des livres et des jeunes (1978-1995), DLDJ, ont insisté régulièrement sur la contribution des bibliothécaires à la lecture des jeunes en présentant des activités d'animation imaginées pour susciter le goût de la lecture et son maintien jusqu'à l'âge adulte.

Dans ces périodiques, l'espace éditorial consacré aux bibliothèques informe sur l'état des lieux, évoque longuement les activités d'animation du livre et de la lecture organisées par des spécialistes du livre; ils soulignent à maintes reprises le travail des bibliothèques qui se sont démarquées par des projets spéciaux, des orientations spécifiques, voire des amé- nagements physiques et un environnement qui tiennent compte des jeunes et de leurs lectures.

Sous différents angles, l'ensemble des perspectives abordées par ces deux revues constitue le champ des représentations de cette presse spécialisée en littérature de jeunesse. Elles traitent des principes sous-jacents qui déterminent toute intervention reliée à la lecture inscrite dans notre société comme acte culturel et social d'envergure. En somme, elles répondent aux questions suivantes: Que faire lire aux jeunes? Quand le faire? Comment intervenir auprès des très petits, des plus grands? Où intervenir? SeIon quels principes et quelles modalités? À l'aide de quelles techniques d'animation? À quelle fréquence?

Au passage, la revue $D L D J$ constate l'état de dégradation des bibliothèques scolaires et conséquemment de ses retombées sur la lecture des jeunes. À l'occasion, les deux revues soulignent les politiques culturelles qui régissent et orientent les bibliothèques, mentionnent les rapports d'enquête, les commissions d'étude ou les subventions allouées par les instances ministérielles pour leur fonctionnement.

À l'automne 1980, $D L D J$ consacre un numéro quasi entier sur le rôle des bibliothèques en insistant sur la place et l'importance du documentaire dans la formation des jeunes à la lecture, autant que sur l'aptitude de ces derniers à les utiliser adéquatement. À cette fin, la revue présente des réalisations d'aménagement d'espace et d'animation du livre et de la lecture dans différentes régions du Québec: la rive-sud de Montréal, Montréal et Québec.

Près de quinze ans plus tard, la même revue, qui s'était surtout préoccupée du documentaire au secondaire, met en évidence les avantages de l'exploitation de la bande dessinée dans ce même milieu d'enseignement. Lurelu s'intéresse autant à la bibliothèque idéale des jeunes québécois, aux bibliothèques 
municipales (1984, 1989), à la bibliothèque scolaire en milieu pluriethnique (1987) qu'à la bibliothèque en milieu hospitalier (1983).

Dans les deux revues, les articles et les chroniques consacrées aux bibliothèques publiques apparaissent en moins grand nombre à la fin des années 1980 . Sans doute est-ce imputable au fait que le Québec est désormais mieux doté en infrastructures, bien qu'à la même époque, les collections s'appauvrissent considérablement, tout comme d'ailleurs, le matériel documentaire.

Dans les revues professionnelles recensées, le thème des bibliothèques se greffe à celui de la lecture des jeunes. Avec des intensités différentes, Lurelu et DLDJ consacrent, selon les périodes analysées [(1977-1987) et (1988-1998)], des espaces éditoriaux variables. Pour la première période, Lurelu publie moins d'une quinzaine d'articles consacrés aux bibliothèques scolaires et publiques alors que $D L D J$ en publie un peu moins; les articles sont répartis aussi bien entre les bibliothèques scolaires de niveau secondaire qu'entre les bibliothèques publiques et les bibliothèques centrales de prêt $(B C P)$ devenues depuis, les Centres régionaux de services aux bibliothèques publiques (CRSBP). Plusieurs fois, les périodiques constatent que les institutions scolaires et publiques ne peuvent jouer leur rôle pédagogique, faute de collections suffisantes, appropriées et diversifiées.

À ce premier ensemble, s'ajoutent des articles consacrés aux responsables de bibliothèques pour la jeunesse. ${ }^{1}$ Ces derniers estiment que «l'intérêt que l'élève manifeste pour la lecture est en proportion de la motivation reçue du professeur et de l'animateur de la bibliothèque».

\section{Les bibliothèques scolaires}

Comme le rôle fondamental de la bibliothèque scolaire se joue dans un environnement éducatif, au fil des décennies, les auteurs brossent l'état des lieux, décrivent des projets spécifiques, identifient le matériel de lecture disponible ou apprécié par les jeunes selon l'ordre d'enseignement, déplorent la pauvreté des bibliothèques de la Commisssion des écoles catholiques de Montréal (CÉCM), l'usure des collections, l'absence de nouveautés, la formation carencée des responsables. Dans la première décennie, les articles décrivent une situation alarmante, alors que ceux de la décennie suivante insistent plus volontiers sur les projets dynamiques et novateurs mis sur pied par des bibliothécaires, du personnel de la direction d'école ou des parents.

Ainsi, Minassian $(1978,5)$ note que si la plupart des écoles ont une bibliothèque scolaire, l'impression qui s'en dégage est celle « d'une accumulation de livres» répartis entre quatre blocs: les livres d'images pour les plus jeunes, les documentaires, certaines séries de bandes dessinées, la file ininterrompue des romans de série (Le Club des cinq, Le Clan des sept, etc.) "plutôt que de bibliothèques planifiées pédagogiquement". À ce relevé s'ajoute une mince production éditoriale québécoise contemporaine désuète. Dans ce contexte, l'auteure signale qu'il suffirait d'une décision administrative pour modifier la situation en faveur de l'édition québécoise pour les jeunes.

Parmi les raisons invoquées pour expliquer la pénurie de ressources matérielles des bibliothèques scolaires de la CÉCM, il y a la destruction du catalogue central du Bureau des bibliothèques scolaires «colonne vertébrale d'un service efficace et intelligent aux étudiants de la CÉCM» (Bélisle 1987, 31). Le Comité d'étude sur les bibliothèques scolaires québécoises, lors de la remise de son rapport au ministre Ryan, fait état de la désuétude et de la pauvreté du réseau, de la régression progressive des acquisitions depuis 1975, de la carence marquée

dans la formation et le perfectionnement des responsables, de l'existence d'un fonds documentaire périmé, et, surtout, souligne une très faible exploitation pédagogique. Le groupe de travail reprend à son compte les cinquante-trois recommandations issues des organismes consultés et propose au gouvernement un vaste plan de redressement et de développement. (Tétrault 1989, 25)

En dépit de l'état alarmant constaté à la Commission des écoles catholiques de Verdun (CÉCV), quatre écoles primaires bénéficient d'un service d'animation de leurs bibliothèques grâce aux budgets accordés aux milieux défavorisés par le Conseil scolaire de l'île de Montréal. Bernier et Tranchant-Hervé (1986), les animateurs engagés, œuvrent dans des bibliothèques bien aménagées bien que sous-utilisées. Par ailleurs, sur la RiveSud, dans les écoles de la Commission scolaire Saint-Exupéry, Jacques Sénéchal, mandaté pour procéder à l'animation pédagogique, note que la bibliothèque scolaire est un milieu de vie plus près du milieu naturel de l'enfant. Denis $(1981,18)$ constate l'« effort de consolidation du triangle classe-bibliothèquemaison".

La bibliothécaire de l'école primaire Saint-Jean-Baptiste (CÉCM), située au coeur du Plateau Mont-Royal, se prononce sur la mission de la bibliothèque à l'école primaire; elle est, à son avis, de "donner le goût de lire sans l'imposer" (Beauchesne 1987, 38). Pour Rachel Boisvert, la bibliothèque est un lieu non seulement d'animation pour donner le goût aux enfants de lire en français, mais aussi un lieu de formation documentaire.

À l'école Laurendeau-Dunton à LaSalle, la bibliothèque était sous-utilisée (5 000 livres); le directeur adjoint, Yves Dubuc, proposa à ses 24 enseignants de lui redonner vie en privilégiant le savoirlire, en augmentant et en soutenant la motivation à la lecture, en faisant de la bibliothèque et des coins de lecture, un petit centre culturel. (Hardy 1978b)

En procédant de la sorte, le personnel scolaire a revalorisé la bibliothèque aux yeux des élèves et des parents. À cette fin, il fut proposé différentes techniques d'expression (mime, jeu, marionnettes) reliées constamment au livre. À ce projet de recherche et de développement pédagogique se sont associés un parrain et une marraine: Guy Boulizon et Bernadette Renaud, auteurs de roman pour les jeunes; ils rencontrent les élèves et leur font connaître les livres québécois qui leur sont destinés: albums, romans, contes, légendes.

\footnotetext{
1. «Notre revue dialogue avec des responsables de bibliothèques pour la jeunesse ". Des livres et des jeunes 3 (7) 1980: 29-32.
} 
Construite en 1973, l'école Gentilly de Longueuil a vécu une expérience d'animation du livre et de la lecture en milieu scolaire non traditionnel, grâce à Denise St-Denis, parent responsable du projet, qui a réuni une dizaine de parents (Hardy 1999a). II s'agissait de donner le goût de la lecture aux enfants; faire de la bibliothèque un instrument utile et accessible afin de permettre aux enfants de se documenter, de savoir utiliser des encyclopédies, des dictionnaires, de chercher les livres sur les rayons, etc... Cette initiative a eu l'heur de dépoussiérer l'image que la population se faisait de leur bibliothèque.

À Outremont, à l'école alternative Querbes, Lucille Richard, responsable d'un groupe multiâges déclare: "Nous n'avons pas besoin de suivre un programme de lecture: les enfants lisent volontiers" (Hardy 1979a, 17). Dans cette école à pédagogie ouverte, la bibliothèque de l'école est sous la responsabilité des parents; ils aident les enfants dans leur recherche et leur facilitent l'accès à la bibliothèque. De plus, chaque local de classe dispose d'un coin pour la lecture. Dictionnaires et encyclopédies côtoient romans, contes, albums et jeux d'apprentissage à la lecture. En somme, la lecture, située au coeur des apprentissages scolaires, prend ici sens et signification, grâce à l'aménagement de coins pour la lecture jumelés à un programme d'animation de la bibliothèque scolaire.

Dans un contexte de changements sociaux à l'échelle planétaire, Mareuil (1980) insiste sur le rôle des bibliothèques à l'école secondaire, rôle qui consiste à développer l'aptitude des élèves à utiliser l'information et la documentation. À Magog en Estrie, en juin 1981, Gaétan Giguère (1982), après avoir dressé un inventaire minutieux des 29808 volumes disponibles à la polyvalente La Ruche, déplore la sous-utilisation du matériel imprimé et audiovisuel mis à la disposition des jeunes et des enseignants. En guise d'exemple, l'auteur constate que si les encyclopédies constituent une mine d'or pour les recherches au secondaire, «c'est le tiers-monde de nos bibliothèques par leur sous-utilisation " II note que parmi les cinquante-sept encyclopédies, seulement trois ou quatre servent régulièrement.
Croteau (1981) constate que si la section des documentaires des polyvalentes de la Commission scolaire catholique de Sherbrooke (CSCS) est bien garnie, les documents relatifs à des techniques ou à des métiers sont non seulement rares, mais présentent des normes autres que québécoises. L'auteur suggère de remédier aux problèmes afin que les usagers ne soient plus pénalisés par un matériel inadéquat.

Enfin, à la mi-décembre 1990, Therriault (1994) note que dans toutes les bibliothèques des écoles secondaires, il existe un rayon réservé aux bandes dessinées, la plupart du temps vide, car les albums repartent aussitôt qu'ils arrivent. N'y aurait-il pas lieu, se demande l'auteure, de profiter de cet engouement pour inscrire la créativité au centre des apprentissages scolaires?

En bref, les deux revues recensées donnent l'heure juste quant à l'état des collections des bibliothèques scolaires au primaire et au secondaire. Elles constatent les nombreux efforts consentis par les bibliothécaires (ou ce qui en tient lieu) pour susciter chez les jeunes le goût de la lecture - par l'organisation de programmes d'animation appropriés et dynamiques - et ce, en dépit de moyens matériels et financiers réduits.

\section{Les bibliothécaires scolaires}

Les revues analysées insistent sur le rôle des bibliothécaires; elles sont perçues comme les «facilitatrices» et les animatrices de la lecture publique en milieu scolaire. Minassian (1978) note que si la plupart des écoles ont une bibliothèque scolaire, peu d'entre elles ont une bibliothécaire. ${ }^{2}$ En fait, ce sont généralement les professeurs qui, une fois par semaine, font office de bibliothécaires avec l'aide ou non de parents bénévoles.

\section{Les bibliothèques publiques}

Plus de dix ans avant que la ministre de la Culture et des Communications, Louise Beaudoin, publie sa politique du livre et de la lecture et consacre un chapitre à la coopération et à l'amélioration des bibliothèques publiques, soit en 1987, paraissaient les résultats de la Commission d'étude sur les bibliothèques publi- ques présidée par Philippe Sauvageau sous le titre: Les bibliothèques publiques: une responsabilité à partager. (Commission d'étude... 1987)

Hélène Charbonneau, responsable des Bibliothèques des jeunes de la ville de Montréal, mentionnait alors que «l'une des premières préoccupations d'une bibliothèque est de rejoindre un public qui n'est pas, a priori, très intéressé par le livre. Notre but n'est pas de faire lire un maximum de livres par l'enfant, mais de le préparer à devenir un bon lecteur adulte, l'aider à se développer, à acquérir une personnalité plus équilibrée" (Hardy 1978a). En ce cas, les techniques d'animation servent d'appât pour attirer les jeunes à la bibliothèque. D'après Charbonneau, s'ils ne sont pas attirés par la lecture, ils viennent alors pour autre chose (jeux, films, etc.); peu à peu, selon la bibliothécaire, ils prendront l'habitude de lire. Pour cela, la bibliothèque doit être accueillante et ses méthodes très souples. À cette époque, Hélène Charbonneau affirmait que l'animation par excellence était incontestablement «L'heure du conte ", carrefour de nombreuses animations. Ainsi, pour que l'enfant n'associe pas uniquement le livre à l'école, mais bien à sa vie de tous les jours, les bibliothèques publiques de la ville de Montréal diversifient leurs activités et s'impliquent dans la vie de quartier en participant aux événements qui s'y déroulent.

Avec une pointe de nostalgie, Camille Bricault (1985), désormais bibliothécaire à Saint-Jean-sur-le Richelieu, relate les nombreuses activités d'animation offertes à la section jeunesse de la bibliothèque centrale d'Ottawa: accueil quotidien de groupes d'enfants, heure du conte, constitution de listes sélectives dressées par le personnel des bibliothèques jeunesse, ateliers, etc.

En regard de sa mission sociale, la bibliothèque publique de la capitale canadienne (O'Grady 1980) a mis sur pied un triple programme d'animation de la

2. Selon Lorraine Pagé, présidente de la Centrale de l'enseignement du Québec (CEQ): " Il ne reste plus que 42 bibliothécaires dans les écoles du Québec [...]. Depuis une dizaine d'années, 75\% des postes de bibliothécaires ont été supprimés. " (La Presse, 21 mars, A 14) 
lecture destiné aux petits et aux plus grands. Pour les enfants d'âge préscolaire, une liste intitulée Tout premiers albums, mise à la disposition des parents d'enfants de 12 à 30 mois, facilite une première initiation au livre. Pour les enfants d'âge scolaire, de nombreuses activités d'animation accompagnées de livres de science-fiction, de romans policiers, de poésie, etc., visent à encourager les jeunes à explorer d'autres genres et d'autres livres que les bandes dessinées. De plus, les clubs de lecture, "Détectives dynamiques", programme créé par la bibliothèque publique de Richmond en Colombie-Britannique, remportent dans la capitale nationale un franc succès auprès des 8 à 12 ans.

Ce travail d'animation se poursuit auprès des adultes. Six fois par année, des ateliers et des causeries ont lieu pour les bibliothécaires et le personnel de soutien. Sur demande, des ateliers à thèmes divers (fantaisie et science-fiction, littérature de jeunesse et problèmes contemporains, albums pour les 3 à 7 ans, livres pour les non-lecteurs, mini bibliothèques à domicile), présentés à des associations d'enseignants et à des centres communautaires, ont connu un succès inestimable.

À Québec, en 1980, s'ouvre la bibliothèque Saint-Jean-Baptiste, succursale de la Bibliothèque de Québec, aménagée à l'intérieur de l'église anglicane StMatthiew. La population du quartier a accès à une collection de plus de 10000 volumes et à plus d'une trentaine de périodiques. Les enfants du quartier ont été privilégiés, car une section complète de la bibliothèque leur a été réservée, modifiant à court terme les habitudes de la lecture publique du quartier.

À Saint-Léonard (Latreille-Huvelin $1979,7)$, la bibliothèque publique dispose d'une section Jeunesse qui «poursuit des objectifs bien définis: l'accès libre et gratuit à la documentation et aux ouvrages choisis en fonction des besoins des jeunes; la préparation à long terme de lecteurs adultes; la libre participation aux activités culturelles pensées et réalisées par et pour les jeunes; le développement personnel des jeunes considérés comme citoyens à part entière». Dans cet esprit, la bibliothèque offre différents services: prêt de documents, référence et aide aux lec- teurs, services aux groupes, animation de la lecture. Par exemple, l'aide aux lecteurs, primordiale dans une bibliothèque pour enfants, les initie principalement à la localisation des documents et à l'utilisation du fichier. Par ailleurs, les activités reliées à l'animation de la lecture regroupent l'heure du conte (3-5 ans, 6-10 ans), la rédaction d'un journal, une thémathèque, un théâtre, des spectacles de marionnettes, des expositions, des rencontres avec des auteurs, des concoursvacances.

En 1980, à Brossard, l'aménagement d'un nouveau local par la Bibliothèque municipale intègre les services offerts aux jeunes à l'ensemble de ceux de la bibliothèque. Yvon-André Lacroix (1980), alors directeur, décrit les services offerts aux 12 ans et moins: heure du conte, rencontres d'écrivains, projections de films, etc. Pour accrocher les jeunes, l'espace qui leur est alloué est attrayant; des présentoirs sont garnis de parutions relatives à leurs intérêts et sur les événements de la saison, la décoration est variée et colorée et les murs présentent des affiches appréciées et recherchées par les jeunes.

C'est à la suite des visites scolaires un moyen efficace de mettre les jeunes en contact avec la bibliothèque et les livres, et surtout de leur donner le goût d'y revenir et de lire - que la bibliothèque de Brossard recrute plusieurs de ses nouveaux abonnés; ces visites sont évaluées à 1500 ou 2000 élèves par an. La bibliothécaire et responsable de la section Jeunesse de cette bibliothèque de la RiveSud, Danielle Champagne-Boulais (Denis 1983, 28) décrit les rapports entretenus entre la bibliothèque de Brossard et les écoles. Les moyens utilisés pour susciter le goût de lire sont variés: la présentation d'une sélection de livres selon le groupe d'âge, le milieu et les centres d'intérêt; une brève présentation des nouveaux titres reçus; la présentation des livres d'un auteur, d'un illustrateur ou d'une collection; une histoire que l'on raconte; un exposé sur la littérature québécoise pour les jeunes. En somme, le but est simple, que “ les jeunes aient une image positive de la bibliothèque et de la lecture si nous voulons qu'à l'âge adulte ils lisent ".

À la fin de la décennie quatre-vingt, Christiane Charette constate une amélio- ration $\mathrm{du}$ rendement des bibliothèques publiques au Québec ainsi qu'une évolution caractérisée par une amélioration de leurs collections, la diversité des documents, mais aussi l'élargissement des services offerts. L'auteure note que lors des agrandissements ou des constructions, on s'est efforcé de donner au secteur jeunesse un cachet spécial.

Durant les années 1980 à 1985, ily a eu des subventions pour la construction et l'agrandissement de bibliotbèques. Ce programme d'aide à la création de bibliotbèques n'a jamais aussi bien fonctionné qu'à cette époque. Il y a eu beaucoup de projets dans tout le Québec. C'est ainsi qu'on a vu plusieurs nouvelles bibliothèques, un peu partout». (Charrette 1989,19 )

À ces transformations s'ajoutent les Bibliothèques centrales de prêts ( $B C P$ ), devenues les Centres régionaux de service aux bibliothèques publiques (CRSBP), conçues pour les 800 municipalités ayant moins de 5000 habitants; elles sont subventionnées de $70 \%$ à $80 \%$ par le gouvernement provincial. Le défi de ces organismes régionaux est de desservir un territoire deux fois et demi plus grand que celui de la France et de maintenir des bibliothèques publiques dans les municipalités québécoises à faible densité de population. Ces corporations privées à but non lucratif, formées à la demande des représentants d'une région et à l'instigation du ministère des Affaires culturelles, ont pour mission spécifique d'établir et de maintenir les structures de développement et de fonctionnement d'un réseau de bibliothèques publiques à l'intérieur d'une région donnée; elles assurent ainsi l'accès à la lecture, à la documentation et à l'information sous toutes ses formes. Avec leurs collections itinérantes, échangées périodiquement, elles offrent à leurs clients des ouvrages de fiction et des documentaires pratiques et encouragent les bibliothèques affiliées à s'associer à leur développement à l'aide du mensuel Le bon choix publié depuis janvier 1989.

Dans les Laurentides, la Bibliothèque centrale de prêts (Picard-Pilon 1986) avec ses $18000 \mathrm{~km}^{2}$ de territoire, ses 64 bibliothèques affiliées et ses 300 bénévoles, a fait vivre à 2280 enfants de la troisième à 
la sixième année, de janvier à mai 1986, de riches activités de lecture; Yves Beauchesne, chargé de projet, était secondé de 78 bénévoles.

Parallèlement à ces modifications, les bibliothèques ont développé de nouveaux services pour les jeunes, grâce à l'édition québécoise et européenne enrichie désormais de livres et de livres-jeux plus attrayants et plus nombreux. Comme l'un des objectifs premiers des bibliothèques publiques est de développer le goût de la lecture chez les jeunes, les programmes d'animation offrent de nombreuses activités, telles les visites dans les classes, en plus de développer une collection de titres adaptés à une jeune clientèle pluriethnique ou handicapée. En 1982, la Bibliothèque de Montréal a accueilli 82678 nouveaux abonnés dont 31774 enfants; elle a prêté au cours de la même année 2396623 documents dont 981789 livres aux enfants. Les 2006622 documents (Soulières 1984) de la bibliothèque (périodiques, journaux, publications officielles, enregistrements sonores, vidéocassettes, films en $16 \mathrm{~mm}$, diapositives, microdocuments) sont répartis parmi les 22 bibliothèques de quartier et le bibliobus.

À Outremont, faute d'une bibliothèque municipale adéquate pour les jeunes, le comité-bibliothèque a organisé la première Kermesse-Soleil, le 13 juin 1987 au parc Beaubien (Gagnon 1987). Les objectifs poursuivis étaient de sensibiliser les jeunes au plaisir de la lecture et au respect des livres, et ce, en les impliquant dans une démarche active. Lors de cet événement, l'activité d'animation du livre et de la lecture a connu un franc succès.

Selon Monique Poulin (1983), en milieu hospitalier, la bibliothèque ne tient malheureusement pas lieu de priorité. Bien que l'hôpital Sainte-Justine possède 8000 livres pour enfants, elle ne peut offrir d'activités centrées sur le livre de jeunesse, faute de bibliothécaire spécialisée. Afin de parer à cet état de fait, la bibliothèque de la ville de Montréal vient à la rescousse de certains de ces hôpitaux comme l'hôpital Marie-Enfant - en lui fournissant 750 livres neufs appartenant à tous les genres littéraires: albums, ro- mans, documentaires, recueils de poésie...

En bref, ce tour d'horizon nous permet de constater que la presse spécialisée en littérature de jeunesse a souligné, à différents moments de sa parution, les projets d'animation réalisés par les bibliothèques publiques pour attirer et fidéliser une jeune clientèle lectrice.

\section{Le personnel spécialisé des \\ bibliothèques publiques: les bibliothécaires}

À la fin des années 1970, la coordonnatrice des services aux enfants à la Bibliothèque de Montréal, Hélène Charbonneau (1980), établissait la bibliothèque idéale des jeunes québécois à partir des besoins des jeunes. Elle soulignait également le rôle des parents; à son avis, la fréquentation des bibliothèques publiques et scolaires ne suffisait pas toujours à donner le goût de lire. L'auteure insistait sur le livre juvénile et décrivait l'édition québécoise pour jeunes publics sous le label d'excellence de cinq maisons: Fides et sa collection «Le Goéland»; Leméac et sa collection «Littérature québécoise »; Héritage et la collection «Pour lire avec toi»; Paulines avec sa revue «Vidéo-Presse» et sa collection « Jeunesse-Pop » et finalement la jeune et dynamique maison, les Éditions de la courte échelle fondée en 1978.

Grâce à une entrevue réalisée par Daniel Sernine, le travail de bibliothécaires chevronnées comme celui d'Hélène Charbonneau est mis en lumière. Pendant plus de 30 ans, cette professionnelle du livre est intervenue auprès des jeunes dans le cadre de ses activités à la Bibliothèque de Montréal. À ses débuts, en 1953, il n'y avait qu'une demi-douzaine de bibliothèques de quartier alors qu'en 1987 , on en trouvait 23 , incluant le bibliobus.

Pendant cette période, la Ville de Montréal compilait des bibliographies consacrées au livre québécois pour la jeunesse comme En panne de livres. En 1972 , suite à sa nomination au poste de chef des bibliothèques pour enfants, Hélène Charbonneau aidait les bibliothécaires à mieux connaître la littérature de jeunesse. Grâce à la complicité de biblio- thécaires comme Christiane Charette, Michèle Gélinas, Ginette Guindon et Marie Pilon, elle s'engageait dans la sélection d'ouvrages pour éveiller les enfants aux livres, les guider pour qu'ils puissent élargir et varier leurs lectures. Cela donna lieu au répertoire Livres en langue française pour les jeunes.

C'est en 1981 que Michèle Gélinas, bibliothécaire à la bibliothèque CentraleEnfants, suggère des activités d'animation culturelle dans une bibliothèque publique. Pour l'heure du conte, elle propose d'accompagner cette activité d'un support audiovisuel (diapositives, feutrine, marottes...), de créer une ambiance propice à la lecture et de choisir un thème. Elle laisse une large place aux clubs de lecture fondés par Hélène Charbonneau en 1960. Cette activité vise à amener l'enfant à lire des nouveautés ou des ouvrages parmi les collections moins récentes. «Certains titres, moins attrayants, demeurent sur les rayons. Et pourtant, ils méritent d'être lus » (Gélinas 1981, 19). Le club de lecture, destiné aux enfants de 1 à 7 ans et aux 12 à 16 ans, est l'occasion rêvée de faire connaître au moins 25 titres par groupe d'âge. Pour que l'entreprise réussisse, il est suggéré de présenter un vaste choix de livres, de ne pas limiter ce choix à des thèmes trop précis, de laisser les jeunes jouer pour leur permettre d'apprivoiser la lecture.

Dans le but de faire connaître et aimer le livre, la bibliothèque Shamrock (Hardy 1979b) de la ville de Montréal a mis sur pied, en 1979, le «Théâtre des livres vivants " pour rafraîchir la formule du livre de lecture pour les 8 à 12 ans. Cette expérience porte ses fruits, car les livres présentés par une marionnette sortent plus souvent des rayons de la bibliothèque. En outre, la mise en scène stimule la créativité du jeune.

\section{En conclusion}

Malgré les manques constatés autant dans les bibliothèques publiques que scolaires (collections insuffisantes, acquisition de ressources documentaires réduites, formation carencée du personnel bénévole), les jeunes lisent désormais plus que leurs aînés (Chouinard 1998). Cet état de fait est imputable aux bibliothécaires qui, inlassablement depuis 
25 ans, ont mis sur pied de nombreuses activités d'animation de la lecture et du livre, généralement de concert avec les établissements scolaires et, faut-il le répéter, le bénévolat de centaines de parents. Comme le souligne Cécile Gagnon (1987, 31): "Dans les milieux des bibliothèques, les activités d'animation sont depuis longtemps florissantes. Elles amènent de nouveaux publics, augmentent l'intérêt des usagers habituels, font connaître toutes sortes de documentation, d'information, de divertissement, et aussi - ce n'est pas négligeable - rapprochent les créateurs de leurs publics".

Tout au long de ces années, Lurelu et $D L D J$ ont beaucoup insisté sur le travail d'animation réalisé, ici et là au Québec et hors de la province, pour développer le goût et le plaisir de la lecture chez les toutpetits et chez les plus grands, par les bibliothèques scolaires, publiques et les BCP (Tétrault 1980). L'importance de ces dernières est perceptible eu égard à la promotion, à la diffusion et à la conservation du patrimoine. Les nombreux témoignages de cette presse ont contribué à convaincre les prescripteurs ( $\mathrm{pa}-$ rents, bibliothécaires, libraires, enseignants et enseignantes) d'intervenir très tôt auprès des jeunes, de leur présenter la lecture de manière attrayante en leur suggérant différentes façons de donner le goût de lire.

En somme, les revues, constantes dans leur propos sur les bibliothèques, ont écrit sous différents modes l'importance d'encourager, très tôt, chez l'enfant des habitudes de lecture permanentes et tenaces, de nourrir cet appétit en ayant en tête la mission sociale des uns et des autres à l'éveil, à la croissance et au maintien de la lecture chez les enfants et les jeunes. À cet égard, les périodiques ont rempli fidèlement leur rôle en encourageant les nombreuses initiatives réalisées sur le territoire québécois et, à l'occasion, hors Québec. Le travail des bibliothécaires scolaires et publiques a été, à maintes occasions, décrit, souligné et louangé.

Sans cette contribution exceptionnelle de la presse professionnelle, le discours sur la lecture des jeunes, centré sur le plaisr de lire "pour la vie ", n'aurait sans doute jamais connu l'impact qu'on lui re- connaît en cette fin de siècle et de millénaire, soit que les jeunes lisent plus que les adultes, du moins au Québec. En fait, ces revues ont amplement supporté la lecture publique en soulignant ses bienfaits auprès des jeunes et de leurs parents, en suggérant de très nombreux moyens de rendre le livre accessible dès la petite enfance, puis en soutenant cet intérêt jusqu'à l'âge adulte.

On peut penser que ces revues ont servi en quelque sorte de "chien de garde" de la lecture publique, alors que les autorités ministérielles semblaient s'en désintéresser en négligeant ces lieux, du moins lors de la distribution des subventions. En dépit du sous-financement chronique des bibliothèques scolaires et publiques, Lurelu et DLDJ ont encouragé le travail souvent obscur de ces centaines d'hommes et de femmes qui ont consacré leur vie professionnelle à la lecture "sous toutes ses formes". Sans les bibliothèques, la lecture des jeunes serait, sans doute, moins florissante qu'elle ne l'est actuellement.

Le panorama, que nous avons peint à larges traits, de la place des bibliothèques publiques et scolaires dans la presse professionnelle québécoise consacrée à la littérature de jeunesse a insisté sur la dimension dynamique, créatrice et innovatrice de ces lieux consacrés à la lecture publique pour le plus grand bénéfice des petits, des jeunes et des plus grands.

\section{Sources consultées}

Beauchesne, Yves. 1987. À la bibliothèque de l'école Saint-Jean-Baptiste. Des livres et des jeunes no 27 : 38-43.

Bélisle, Alvine. 1987. Pour rétablir une certaine vérité. Des livres et des jeunes no 28: 31.

Bernier, Luc et Marie-Bénédicte Tranchant-Hervé 1986. Dix ans d'animation dans les bibliothèques scolaires de Verdun. Des livres et des jeunes no 26: 33-37.

Bricault, Camille. 1985. Lire à la bibliothèque d'Ottawa: une aventure dont vous êtes le héros. Des livres et des jeunes 7 (22): 15-17.

Charbonneau, Hélène. 1980. La bibliothèque idéale des jeunes Québécois. Lurelu 3 (1): 3-7.

Charette, Christiane. 1989. Les années ' 80 dans les bibliothèques publiques. Lurelu 12 (2): 18-19.
Chouinard, Marie-Andrée. 1998. Pour que tous lisent. Le Devoir, samedi le 21 mars et dimanche le 22 mars: D 5.

Commission d'étude sur les bibliothèques publiques. 1987. Lurelu 10 (2): 16-19.

Croteau, Marcellin. 1981. Les documentaires au secondaire. Des livres et des jeunes 3 (9): 15-18.

Denis, Anne. 1981. La bibliothèque scolaire: un milieu de vie. Lurelu 4 (1-2): 18.

1983. Les visites scolaires à la bibliothèque municipale. Lurelu 6 (1): 28

Gagnon, Cécile. 1987. Virel s'amuse au parc Beaubien. Lurelu 10 (2) : 30-31.

Gélinas, Michèle. 1981. Quelques activités d'animation culturelle dans une bibliothèque publique. Des livres et des jeunes 4 (10) : 20-25.

Giguère, Gaétan. 1982. Des documentaires informatifs dans une polyvalente. Des livres et des jeunes 4 (11): 24-26.

Hardy, Diane. 1978a. L'animation dans les bibliothèques publiques de Montréal. Lurelu 1(1): n.p.

1978b. Projet: bibliothèque vivante. Lurelu 1 (3): 15 .

1979a. Deux décennies en milieu scolaire non traditionnel. Lure/u 2 (3): 16-17.

1979b. Le théâtre des livres vivants. Lurelu 2 (1): 7 .

1980. Avoir du plaisir à lire. Lurelu 3 (4): 19

Lacroix, Yvon-André. 1980. Aménagement de la section des enfants: le cas de Brossard. Des livres et des jeunes $3(7): 19-21$.

Latreille-Huvelin, France. 1979. À la bibliothèque publique de Saint-Léonard. Des livres et des jeunes 2 (4) 7-8.

Mareuil, André. 1980. À l'école secondaire, le rôle des bibliothèques. Des livres et des jeunes 3 (7) : 912.

Minassian, Chaké. 1978. Le problème de la lecture et des bibliothèques scolaires. Lurelu 1(2): 3-5.

O'Grady Marina. 1980. Programmes d'animation à la bibliothèque publique d'Ottawa. Des livres et des jeunes $3(7)$ : 13-15.

Picard-Pilon, Louise. 1986. La lecture ne se fait pas qu'avec les yeux! Des livres et des jeunes (2425): 52-53.

Poulin, Monique. 1983. La bibliothèque à l'hôpital. Lurelu $6(2): 26$

Soulières, Robert. 1984. Vite dit. Lurelu 7(1): 27.

Tétrault, Raymond. 1980. Les BCP un service «de pointe \%. Des livres et des jeunes 3 (7) : 23-25.

1989. Les bibliothèques scolaires québécoises... plus que jamais. Des livres et des jeunes (34): 25.

Therriault, Annie. 1994. La bande dessinée au secondaire; pourquoi pas? Des livies et des jeunes (46) : 14-17. 